\title{
Small-size silver nanoparticles stimulate neutrophil oxidative burst through an increase of intracellular calcium levels
}

\author{
MARISA FREITAS $^{1}$, MARIANA LUCAS $^{1}$, ADELAIDE SOUSA ${ }^{1}$, TÂNIA SOARES ${ }^{1}$, \\ DANIELA RIBEIRO $^{1}$, FÉLIX CARVALHO ${ }^{2}$ and EDUARDA FERNANDES ${ }^{1}$ \\ ${ }^{1}$ LAQV, REQUIMTE, Laboratory of Applied Chemistry, Department of Chemical Sciences, \\ and ${ }^{2}$ UCIBIO, REQUIMTE, Laboratory of Toxicology, Department of Biological Sciences, \\ Faculty of Pharmacy, University of Porto, 4050-313 Porto, Portugal
}

Received January 29, 2020; Accepted April 13, 2020

DOI:10.3892/wasj.2020.46

\begin{abstract}
Despite the widespread use of silver nanoparticles (AgNPs) in several daily consumer products, the putative human risks resulting from continuous exposure remain elusive, particularly with what concerns the reactivity of the immune system and the resulting pro-inflammatory effects. Neutrophils are considered one of the first and primary cell types that process systemic nanoparticles, mediating the host inflammatory and immunological response, involving the production of reactive oxygen and nitrogen species. The main objective of the present study was to evaluate the effect of polyvinylpyrrolidone (PVP) and citrate-coated AgNPs of different sizes $(5,10$ and $50 \mathrm{~nm})$ on oxidative burst, calcium levels and the viability of human neutrophils. It was observed that the effects of AgNPs were size and coating-dependent. The citrate-coated and 5-nm-sized AgNPs were the most cytotoxic and potent inducers of neutrophil oxidative burst. It was observed that AgNPs induced an increase in intracellular calcium levels, which are involved in the activation of protein kinase $\mathrm{C}$ (PKC), resulting in the assembly of NADPH oxidase subunits with the subsequent production of reactive oxygen species. This excessive production of reactive oxygen species most probably contributes to the AgNP-induced decrease in neutrophil viability. Overall, the data of the present study contribute to a better understanding of the pro-inflammatory mechanisms associated with the use of AgNPs.
\end{abstract}

Correspondence to: Dr Marisa Freitas or Dr Eduarda Fernandes, LAQV, REQUIMTE, Laboratory of Applied Chemistry, Department of Chemical Sciences, Faculty of Pharmacy, University of Porto, Rua de Jorge Viterbo Ferreira 228, 4050-313 Porto, Portugal

E-mail: marisafreitas@ff.up.pt

E-mail: egracas@ff.up.pt

Key words: silver, neutrophils, calcium, reactive species, nanoparticles

\section{Introduction}

The medicinal properties of silver-based compounds have been known for $>2,000$ years. Since the 19th century, these properties have been associated with their antimicrobial activity. More recently, advancements in the nanoscience and nanotechnology fields have resulted in the development of several consumer products, many of which are routinely used in daily life. Inevitably, researchers are paying attention on metallic nanoparticles due to their increasing microbial resistance against metal ions, antibiotics and the development of resistant strains (1). Among the metallic nanoparticles, particular attention has been paid to silver nanoparticles (AgNPs), which presently correspond to $24 \%$ of the 1,814 products listed in the Nanotechnology Consumer Products Inventory (2). Efforts have been made to explore their attractive properties, allowing their use as antibacterial and anticancer drugs, in diagnostics and optoelectronics, in water disinfection, cosmetics, and other clinical/pharmaceutical applications. The majority of these products are already available for purchase at grocery stores and through the internet $(3,4)$. Moreover, the silver antimicrobial agents can be easily incorporated into several materials, such as plastics and textiles, making them useful in a wide spectrum of applications, maintaining their antimicrobial activity in situ, in which traditional antimicrobial agents would be unstable.

It is well known that nanoparticles can be recognized by the immune system, which may result in the activation of pro-inflammatory pathways (5). The intentional or unintentional human exposure to AgNPs is unavoidable and may also trigger innate immunity responses. The innate immunity is the nonspecific and first line of the body's defense system that plays an essential role in the early recognition of non-self and foreign bodies and subsequent pro-inflammatory response. Among the innate immune response cells, leukocytes, neutrophils constitute the first cells to arrive to the affected local of inflammation where they usually phagocyte and neutralize the invader. This process involves the production of reactive oxygen species (ROS) and reactive nitrogen species (RNS), a process known as oxidative burst. When this production is exacerbated and sustained, it may result in oxidative stress, a condition involved in the development and worsening of 
several diseases $(6,7)$. It is currently accepted that several chemical and physical properties of AgNPs, including their size, shape and surface coatings, directly affect the nanoparticle compatibility with the immune system. In this context, the coating agents, essentially used to stabilize AgNPs, maintaining their specific characteristics, are also responsible for the immunogenic properties (8). Two commonly used coating agents are sodium citrate and polyvinylpyrrolidone (PVP), which impart a negative charge, giving AgNPs a wide appeal for manufacturing and consumer use (9).

Previous studies have reported the pro-inflammatory effects of AgNPs, in which it was demonstrated that such nanoparticles are responsible for an increased number of neutrophils in lungs/bronchoalveolar lavage fluid (9-12). Nevertheless, the literature dealing with the direct interaction of AgNPs with human neutrophils is still limited (13-15), investigating only one particle type with one coating type and/or one size, at a limited dose range. Therefore, the present study examined the effect of 3 different sizes $(5,10$ and $50 \mathrm{~nm})$ of citrate- and PVP-coated AgNPs on oxidative burst, calcium levels and the viability of human neutrophils.

\section{Materials and methods}

Reagents. BioPure PVP and citrate coated-AgNPs (5, 10 and $50 \mathrm{~nm}$ ) were obtained from nanoComposix. The manufacturer characterizes each batch with: Transmission electron microscopy (TEM) to determine size and shape distributions (all the AgNPs present a spherical form) (Table I); UV-visible spectroscopy to measure the optical properties; dynamic light scattering to determine particle hydrodynamic diameter; and zeta potential measurement to determine particle surface charge. BioPure nanoparticles are extensively washed with the suspending solvent to remove residual reactants from the manufacturing process. Mass concentration is determined with inductively coupled plasma mass spectroscopy (ICP-MS). The particles are sterile filtered and tested for endotoxin contamination before delivery. Throughout the study, nanomaterials were stored at $4^{\circ} \mathrm{C}$.

Trypan blue solution, histopaque 1077, histopaque 1119, dihydrorhodamine 123 (DHR), diphenyleneiodonium chloride (DPI), 1-(5-chloronaphthalene-1-sulfonyl)-1H-hexahydro-1,4diazepine hydrochloride (ML-9), 3-[1-[3-(Dimethylamino) propyl]-5-methoxy-1H-indol-3-yl]-4-(1H-indol-3-yl)-1H-pyrrole-2,5dione (Gö6983), RPMI-1640 medium, fetal bovine serum, L-glutamine, penicillin, streptomycin, Dulbecco's phosphatebuffered saline, without calcium chloride and magnesium chloride (PBS) were obtained from Sigma-Aldrich; Merck KGaA. The Annexin V-FLUOS Staining kit was obtained from Roche Diagnostics GmbH. FLUO-4/AM was purchased Life Technologies; Thermo Fisher Scientific, Inc. Vacuum tubes with $\mathrm{K}_{3}$ EDTA were purchased from Vacutainer Systems.

Equipment. Assays were performed in a microplate reader (Synergy HT, BioTek Instruments, Inc.), using colorimetric and fluorimetric detection, and a flow cytometer (Accuri ${ }^{\mathrm{TM}}$ C6, BD Biosciences).

Isolation of human neutrophils. All patient-related procedures and protocols were performed in accordance with the
Declaration of Helsinki and approved by the Ethics Committee of Centro Hospitalar do Porto.

After written informed consent was obtained, venous blood was collected by antecubital venipuncture into $\mathrm{K}_{3}$ EDTA vacuum tubes, in Centro Hospitalar do Porto-Hospital de Santo António blood bank. The isolation of human neutrophils was performed by the density gradient centrifugation method, as previously reported by the authors' research group (16). Briefly, $3 \mathrm{ml}$ of histopaque 1077 were carefully layered on top of $3 \mathrm{ml}$ of histopaque 1119 in a $15 \mathrm{ml}$ polypropylene tube. Subsequently, $4.5 \mathrm{ml}$ of the collected blood were decanted on top of this discontinuous density gradient. The tube was centrifuged at $890 \mathrm{xg}$ for $30 \mathrm{~min}$ at $20^{\circ} \mathrm{C}$. Following centrifugation, the neutrophil pellet was removed using a Pasteur pipette and doubled in volume using PBS; the neutrophils were then centrifuged at $870 \mathrm{x}$ g for $5 \mathrm{~min}$ at $4^{\circ} \mathrm{C}$. The supernatant was decanted and a mixture of $1.25 \mathrm{ml}$ of PBS and $5.25 \mathrm{ml}$ of sterile distilled water was added to the neutrophil pellet to lyse any remaining red blood cells. The tube was gently inverted for $2 \mathrm{~min}$, after which isotonicity was re-established by adding $2.2 \mathrm{ml}$ of $3 \% \mathrm{NaCl}$. This suspension was then submitted to a new centrifugation at $870 \mathrm{x}$ g for $5 \mathrm{~min}$ at $4^{\circ} \mathrm{C}$ after which the supernatant was decanted and the neutrophil pellet resuspended in RPMI-1640 incubation medium [(pH 7.4) supplemented with $10 \%$ fetal bovine serum, $2 \mathrm{mM}$ L-glutamine, $100 \mathrm{U} / \mathrm{ml}$ penicillin and $0.1 \mathrm{mg} / \mathrm{ml}$ streptomycin]. Cell viability (>98\%) and cell yield (number of cells $/ \mathrm{ml}$ ) were determined by the trypan blue exclusion method using a Neubauer chamber and an optic microscope. The suspension with the isolated neutrophils was kept on ice until use.

Measurement of neutrophil oxidative burst. The measurement of neutrophil oxidative burst was performed by fluorescence, by monitoring the oxidation of DHR to rhodamine 123 by neutrophil-generated ROS (15). Neutrophils $\left(3 \times 10^{6} \mathrm{cells} / \mathrm{ml}\right)$ were incubated in a humidified incubator, at $37^{\circ} \mathrm{C}$, for $2 \mathrm{~h}$ with citrate or PVP-coated AgNPs $(0-50 \mu \mathrm{g} / \mathrm{ml})$, or PVP $(15 \mu \mathrm{g} / \mathrm{ml})$, or citrate $(0.3 \mu \mathrm{g} / \mathrm{ml})$ or with $\mathrm{Ag}^{+}\left(\mathrm{AgNO}_{3}\right)(12 \mu \mathrm{g} / \mathrm{ml})$, followed by DHR $(10 \mu \mathrm{M})$. At the end of this incubation period, cells were centrifuged $\left(400 \mathrm{x} \mathrm{g}\right.$ for $5 \mathrm{~min}$ at $20^{\circ} \mathrm{C}$ ) and the supernatant was discarded. The pellets were resuspended in $300 \mu \mathrm{l}$ of RPMI-1640 medium and the fluorescence was measured using a microplate reader (excitation wavelength, $485 \mathrm{~nm}$; and emission wavelength, $520 \mathrm{~nm}$ ). A control assay (without AgNPs) was always performed in all the experiments.

Involvement of NADPH oxidase in the AgNP-induced neutrophil oxidative burst. The measurement of neutrophil oxidative burst was performed as described above with a few alterations. Neutrophils $\left(3 \times 10^{6}\right.$ cells $\left./ \mathrm{ml}\right)$ were incubated for $10 \mathrm{~min}$ with a NADPH oxidase inhibitor, DPI $(20 \mu \mathrm{M})$, followed by $2 \mathrm{~h}$ of incubation with $5 \mathrm{~nm}$ of citrate and PVP-coated AgNPs $(50 \mu \mathrm{g} / \mathrm{ml})$ and the probe DHR $(10 \mu \mathrm{M})$ at $37^{\circ} \mathrm{C}$. At the end of this incubation period, the protocol used was the same as that described above.

Involvement of protein kinase C (PKC) in the AgNP-induced neutrophil oxidative burst. The measurement of the neutrophil oxidative burst was performed as described above with a few alterations. Neutrophils $\left(3 \times 10^{6}\right.$ cells $\left./ \mathrm{ml}\right)$ were incubated for 
Table I. Diameter measured by TEM and coefficient of variation of the studied AgNPs, according the manufacturer, nanoComposix.

\begin{tabular}{lcc}
\hline & Diameter (nm) & $\begin{array}{c}\text { Coefficient of } \\
\text { variation }(\%)\end{array}$ \\
\hline PVP-coated AgNPs (nm) & & \\
5 & $4.4 \pm 0.9$ & 19.8 \\
10 & $10.1 \pm 1.8$ & 17.5 \\
50 & $50 \pm 4$ & 8.1 \\
Citrate-coated AgNPs (nm) & & \\
5 & $5.2 \pm 0.9$ & 16.4 \\
10 & $9.9 \pm 1.9$ & 18.7 \\
50 & $48 \pm 5$ & 11.2 \\
\hline
\end{tabular}

PVP, polyvinylpyrrolidone; AgNPs, silver nanoparticles.

10 mins with a PKC inhibitor, Gö6983 (5 $\mu \mathrm{M})$, followed by $2 \mathrm{~h}$ of incubation with $5 \mathrm{~nm}$ of citrate and PVP-coated-AgNPs $(50 \mu \mathrm{g} / \mathrm{ml})$ and the probe DHR $(10 \mu \mathrm{M})$ at $37^{\circ} \mathrm{C}$. At the end of this incubation period, the protocol used was the same as that described above.

Measurement of intracellular free calcium levels. The measurement of intracellular calcium levels was performed as previously described by Ribeiro et al (17). Isolated neutrophils $\left(4 \times 10^{6}\right.$ cells $\left./ \mathrm{ml}\right)$ were pre-incubated with FLUO-4/AM $(3 \mu \mathrm{M})$, during $30 \mathrm{~min}$, in a humidified incubator, at $37^{\circ} \mathrm{C}$, followed by the incubation with citrate and PVP-coated AgNPs $(0-50 \mu \mathrm{g} / \mathrm{ml})$ for $2 \mathrm{~h}$. Cells were then centrifuged $(870 \mathrm{x} \mathrm{g}$ for $5 \mathrm{~min}$ at $20^{\circ} \mathrm{C}$ ). The pellet was resuspended in PBS and the cell number readjusted. The monitoring of the intracellular free calcium flux was performed using a microplate reader, at $37^{\circ} \mathrm{C}$, under continuous soft shaking. The excitation and emission wavelengths used were 485 and $590 \mathrm{~nm}$, respectively.

The role of calcium flux in the AgNP-induced neutrophil oxidative burst. To further elucidate the role of calcium flux in the AgNP-induced neutrophil oxidative burst, the store-operated calcium entry (SOCE) broadly investigated inhibitor, ML-9, was used as follows: Neutrophils $\left(3 \times 10^{6}\right.$ cells $\left./ \mathrm{ml}\right)$ were incubated with ML-9 $(100 \mu \mathrm{M})$ for $10 \mathrm{~min}$ and then incubated for $2 \mathrm{~h}$ with $5 \mathrm{~nm}$ of citrate and PVP-coated AgNPs $(50 \mu \mathrm{g} / \mathrm{ml})$, followed by DHR $(10 \mu \mathrm{M})$ at $37^{\circ} \mathrm{C}$. At the end of this incubation period, the protocol used was the same as that described above.

Evaluation of cell death. The evaluation of cell death was analyzed by flow cytometry following simultaneous staining with Annexin $\mathrm{V}$ labeled with fluorescein and propidium iodide, according to a previously described method (18). Neutrophils $\left(1 \times 10^{6}\right.$ cells $\left./ \mathrm{ml}\right)$ were incubated in a humidified incubator, at $37^{\circ} \mathrm{C}$, with citrate- or PVP-coated AgNPs $(0-50 \mu \mathrm{g} / \mathrm{ml})$, or PVP $(15 \mu \mathrm{g} / \mathrm{ml})$, or citrate $(0.3 \mu \mathrm{g} / \mathrm{ml})$ or with $\mathrm{Ag}^{+}\left(\mathrm{AgNO}_{3}\right)(12 \mu \mathrm{g} / \mathrm{ml})$, for $2 \mathrm{~h}$. The commercial Annexin V-FLUOS Staining lit (Roche Diagnostics GmbH) was used according to the manufacturer's instructions.
Fluorescence signals for each sample were collected using a flow cytometer. To restrict the analysis to neutrophils only, a polygon gate was set according to their light scattering properties (in a forward vs. side scatter plot) excluding cell debris and other blood cells. Fluorescence signals for at least 10,000 cells were collected in logarithmic mode and the data were analyzed using C Flow (Accuri) software. The green fluorescence due to Annexin V conjugated with FITC was followed in channel 1 (FL1) and plotted as a histogram of FL1 staining. Fluorescence due to the propidium iodide incorporation was followed in channel 3 (FL3).

Statistical analysis. The GraphPad Prism 6 software was used to calculate all the mean \pm standard deviation of the mean (SEM), (from at least 6 individual experiments, performed in triplicate in each experiment). Statistical comparison between groups was estimated using one-way analysis of variance (ANOVA), followed by the Bonferroni's post hoc test. In all cases, P-values $<0.05$ were considered to indicate statistically significant differences. The results were expressed according to the following equation:

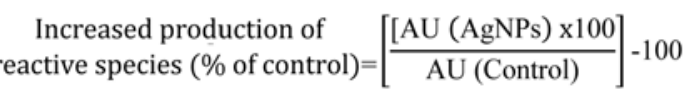

where AU represents arbitrary units, AgNPs represents cells treated with AgNPs and the Control represents untreated cells (without AgNPs).

\section{Results}

Neutrophil oxidative burst. The AgNP-induced neutrophil oxidative burst was measured by DHR. This probe detects total intracellular ROS/RNS, with certain specificity towards hydrogen peroxide $\left(\mathrm{H}_{2} \mathrm{O}_{2}\right)$ and hypochlorous acid $(\mathrm{HOCl})(6)$. As shown in Fig. 1, both the PVP- and citrate-coated AgNPs with sizes of 5 and $10 \mathrm{~nm}$ induced neutrophil oxidative burst in a concentration-dependent manner, while the 50-nm-sized AgNPs had no effect with both coatings. The results also evidenced a higher reactivity for the 5-nm-sized AgNPs (significant effects were observed beginning at $25 \mu \mathrm{g} / \mathrm{ml}$ ) compared to the 10-nm-sized AgNPs (significant effects were only observed at $50 \mu \mathrm{g} / \mathrm{ml}$ ). It was also clear that citrate-coated AgNPs induced greater reactive species production than the PVP-coated AgNPs. None of the coating agents, or $\mathrm{Ag}^{+}$ (in the form of $\mathrm{AgNO}_{3}$ ), per se, induced the production of reactive species (data not shown).

Contribution of NADPH oxidase to the activation of neutrophil oxidative burst by AgNPs. NADPH oxidase is an enzymatic complex responsible for initiating the production of reactive species by human neutrophils. Thus, DPI, an inhibitor of NADPH oxidase, was used to examine the involvement of this enzymatic complex in the AgNP-induced neutrophil oxidative burst. The assays were performed with the 5-nm-sized AgNPs (the nanoparticles that induced the most pronounced effects in the neutrophil oxidative burst). The use of DPI resulted in a decrease in both the PVP- and citrate-coated AgNP-induced neutrophil oxidative burst. The fluorescent signal decreased 

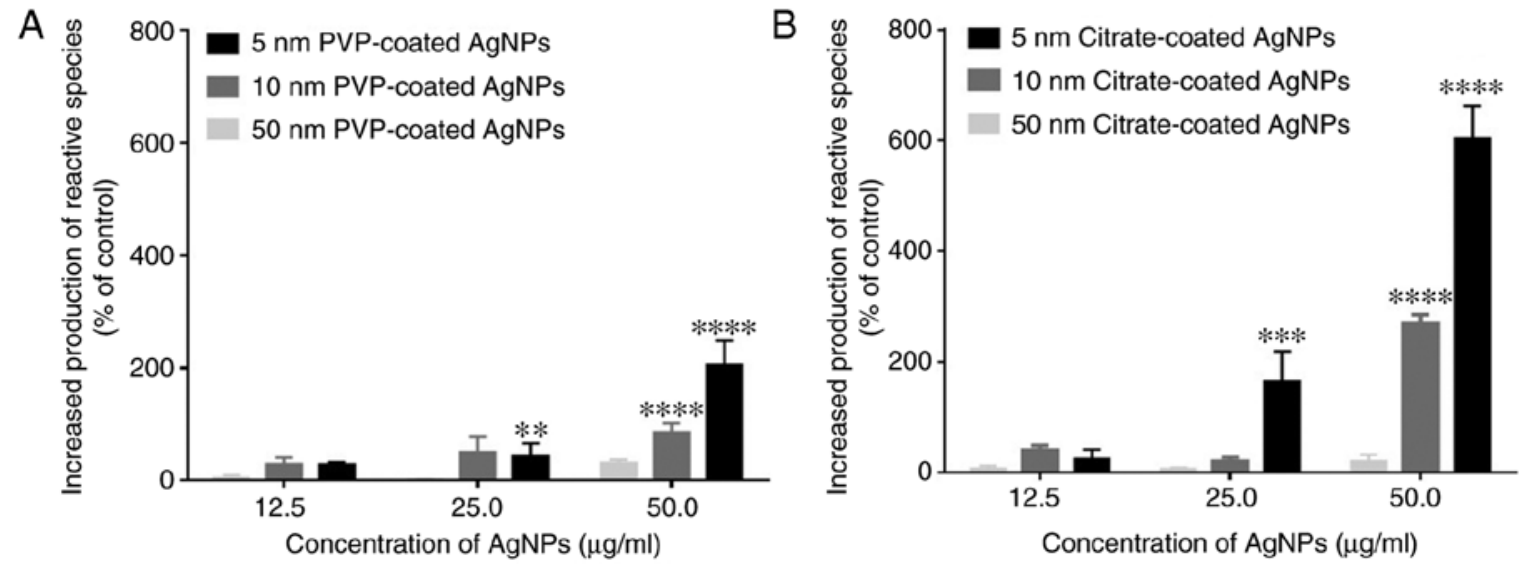

Figure 1. Effect of (A) PVP-coated AgNPs and (B) citrate-coated AgNPs on human neutrophil oxidative burst, measured by DHR. ${ }^{* *} \mathrm{P}<0.01,{ }^{* * *} \mathrm{P}<0.001$, ${ }^{* * * * *} \mathrm{P}<0.0001$, when compared with the control assay (without AgNPs). Values are presented as the means \pm SEM (n $\geq 6$ ). PVP, polyvinylpyrrolidone; AgNPs, silver nanoparticles; DHR, dihydrorhodamine 123.

to values close to the basal levels, which indicated that the AgNPs induced the production of reactive species via NADPH oxidase activation (Fig. 2).

Contribution of PKC to the activation of neutrophil oxidative burst by AgNPs. To analyze the involvement of PKC in the activation of NADPH oxidase by AgNPs, a specific inhibitor of PKC, Gö6983, was used. This inhibitor also prevented the activation of neutrophil oxidative burst by the 5-nm-sized PVP- and citrate-coated AgNPs, indicating that the observed effect was dependent on PKC activation (Fig. 3).

Effect of AgNPs on intracellular free calcium levels. Calcium is a well-known intracellular second messenger with proven involvement in a wide variety of biological processes in human neutrophils. FLUO-4/AM, that belongs to a new group of fluorescent indicators, was used to measure the free cytosolic calcium. At the tested conditions, only the 5-nm-sized citrate-coated AgNPs, at the concentration of $50 \mu \mathrm{g} / \mathrm{ml}$ were able to induce an increase in the intracellular free calcium levels. The PVP-coated AgNPs induced a slight increasing trend that did not achieve statistical significance (Fig. 4).

Involvement of calcium in the activation of neutrophil oxidative burst by AgNPS. In order to determine the role of calcium in the production of reactive species induced by AgNPs, the SOCE inhibitor, ML-9, was used. As shown in Fig. 5, the use of ML-9 decreased the human neutrophil oxidative burst induced by 5-nm-sized citrate- and PVP-coated AgNPs. These results clearly demonstrate the important role of calcium in the effects of AgNPs.

Assessment of neutrophil apoptosis vs. necrosis. To investigate the ability of AgNPs to induce apoptosis and/or necrosis, human neutrophils were exposed during $2 \mathrm{~h}$ to increasing concentrations of PVP- and citrate-coated AgNPs (5, 10 and $50 \mathrm{~nm}$ in size $)$, as well as PVP $(15 \mu \mathrm{g} / \mathrm{ml})$, citrate $(0.3 \mu \mathrm{g} / \mathrm{ml})$ and $\mathrm{Ag}^{+}\left(\mathrm{AgNO}_{3}\right)(12 \mu \mathrm{g} / \mathrm{ml})$. Under the tested experimental conditions, the AgNPs did not induce neutrophil apoptosis, since the number of Annexin $\mathrm{V}$ positive cells did not increase significantly when compared with the control (without AgNPs).

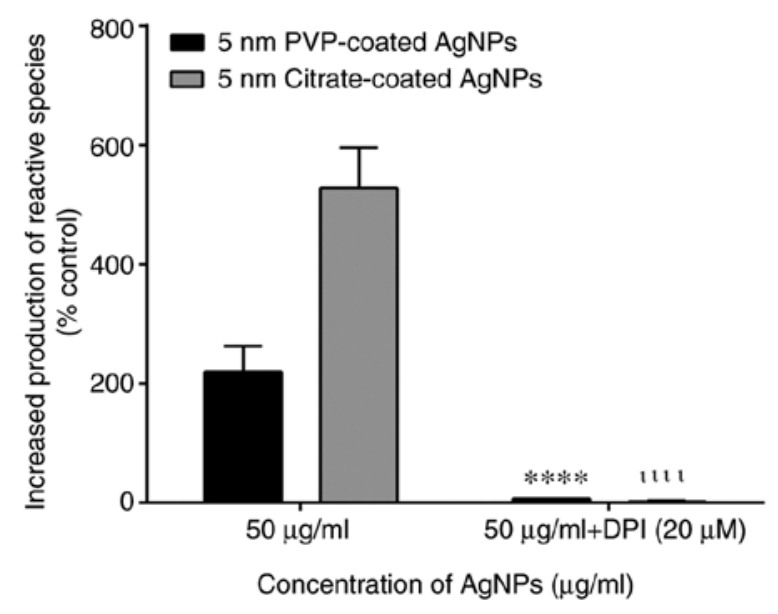

Figure 2. Effect of DPI $(20 \mu \mathrm{M})$ on human neutrophil oxidative burst induced by $5 \mathrm{~nm}$ of PVP- and citrate-coated AgNPs, measured by DHR. ${ }^{* * * *} \mathrm{P}<0.0001$ when compared with PVP-coated AgNPs $(50 \mu \mathrm{g} / \mathrm{ml}, 5 \mathrm{~nm})$. " ${ }^{\text {"u }}<0.0001$ when compared with the citrate-coated AgNPs $(50 \mu \mathrm{g} / \mathrm{ml}, 5 \mathrm{~nm})$. Control, without AgNPs. Values are presented as the means \pm SEM $(n \geq 6)$. DPI, diphenyleneiodonium chloride; DHR, dihydrorhodamine 123; PVP, polyvinylpyrrolidone; AgNPs, silver nanoparticles.

By contrast, the number of PI-positive cells increased in both types of coated AgNPs tested, as it can be seen in the representative flow cytometry plots (Fig. 6A-C). The smallest-sized AgNPs of $5 \mathrm{~nm}$, induced the most potent effect, with $25 \mu \mathrm{g} / \mathrm{ml}$ of the 5-nm-sized of citrate-coated AgNPs being sufficient to induce an increase in the number of necrotic cells (Fig. 6E). Under the tested experimental conditions, PVP, citrate and $\mathrm{Ag}^{+}$, per se, did not influence neutrophil viability (data not shown).

\section{Discussion}

Neutrophils are considered one of the first and primary cell types that process circulating nanoparticles, mediating the host inflammatory and immunological response, which involve the production of reactive species. Although neutrophils play an important role in early stages of inflammation, damage can occur in affected tissues if the stimulus persists (19). The 


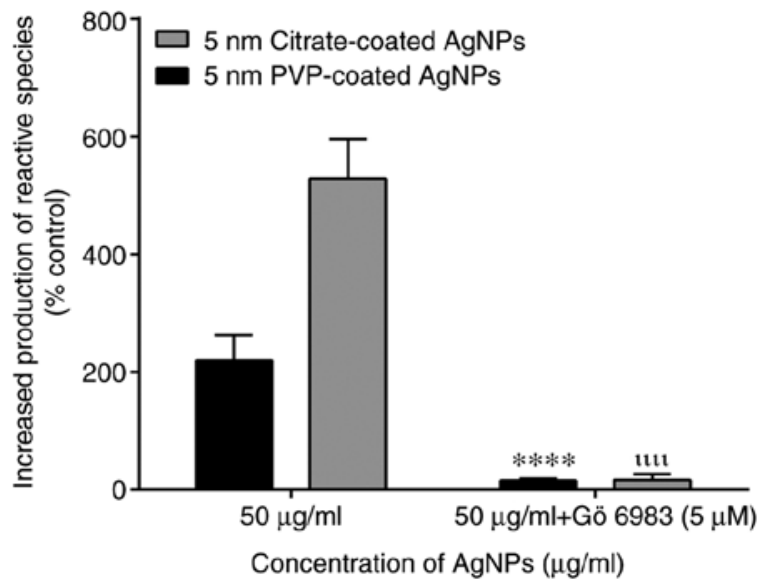

Figure 3. Effect Gö6983 (5 $\mu \mathrm{M})$ on human neutrophil oxidative burst induced by $5 \mathrm{~nm}$ of PVP- and citrate-coated AgNPs, measured by DHR. ${ }^{* * * * *} \mathrm{P}<0.0001$ when compared with the PVP-coated AgNPs $(50 \mu \mathrm{g} / \mathrm{ml}, 5 \mathrm{~nm})$. "'t $\mathrm{P}<0.0001$ when compared with the citrate-coated AgNPs $(50 \mu \mathrm{g} / \mathrm{ml}, 5 \mathrm{~nm})$. Control, without AgNPs. Values are presented as the means \pm SEM $(n \geq 6)$. DHR, dihydrorhodamine 123; PVP, polyvinylpyrrolidone; AgNPs, silver nanoparticles.

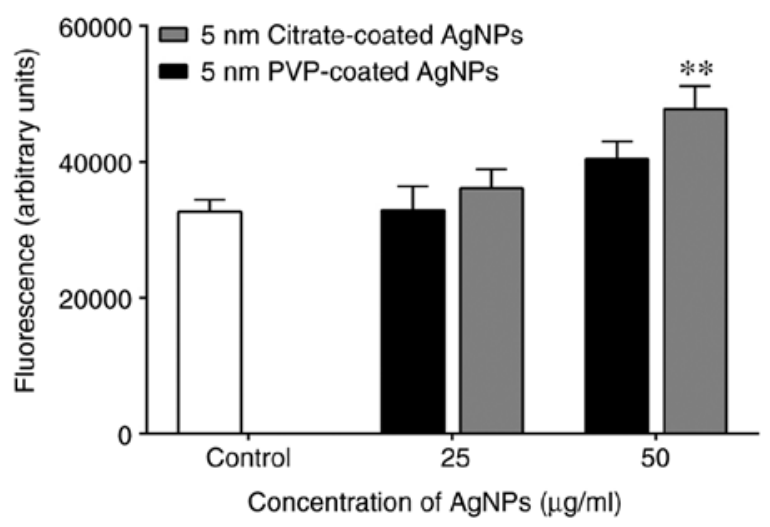

Figure 4. Effect of PVP-and citrate-coated AgNPs on human neutrophil intracellular calcium levels, measured by FLUO-4/AM. ${ }^{* *} \mathrm{P}<0.01$ compared with the control (without AgNPs). Values are presented as the means \pm SEM $(\mathrm{n} \geq 6)$. PVP, polyvinylpyrrolidone; AgNPs, silver nanoparticles.

information available in the literature concerning the interaction of AgNPs with neutrophils is controversial, probably due to the differences found in the experimental conditions, as the cellular model used, the concentrations used, etc. Moreover, given that the $\mathrm{Ag}^{+}$release rate is variable and depends on multiple factors (e.g., AgNPs size and surface area and ambient conditions), it is expected that the citrate- and PVP-coated AgNPs induce different responses. To the best of our knowledge, this is the first study in which PVP- and citrate-coated AgNPs were tested together against human neutrophil main activities. Silva et al (20) instilled Sprague-Dawley rats with AgNPs and concluded that both citrate- and PVP-coated AgNPs induced inflammation, possibly through the increase in neutrophils levels. However, that effect was independent of the coating agent used. By contrast, Seiffert et al (9) intratracheally-administered PVP- and citrate-coated AgNPs to Brown-Norway rats and reported that there was a persistent neutrophilic inflammation more pronounced when citrate-coated AgNPs were used. Herein, the effects of

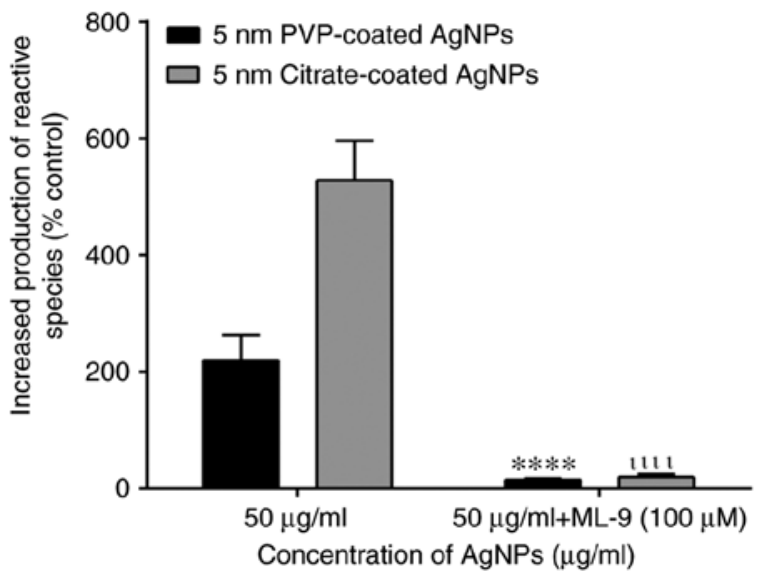

Figure 5. Effect of ML-9 $(100 \mu \mathrm{M})$ on human neutrophil oxidative burst induced by $5 \mathrm{~nm}$ of PVP-and citrate-coated AgNPs, measured by DHR. ${ }^{* * * * *} \mathrm{P}<0.0001$ when compared with the PVP-coated AgNPs $(50 \mu \mathrm{g} / \mathrm{ml}, 5 \mathrm{~nm})$. ${ }^{\text {"'P }}<0.0001$ when compared with the citrate-coated AgNPs $(50 \mu \mathrm{g} / \mathrm{ml}, 5 \mathrm{~nm})$. Control, without AgNPs. Values are presented as the means \pm SEM $(n \geq 6)$. PVP, polyvinylpyrrolidone; AgNPs, silver nanoparticles.

various concentrations of $\mathrm{AgNPs}(5,10$ and $50 \mathrm{~nm}$ ), coated with citrate and PVP on human neutrophils were investigated. The concentrations used in the present study can be attained in individuals following years of exposure, or following acute accidental exposure to AgNPs. As a good example, using exposure data from a AgNPs manufacturing facility, $10 \mu \mathrm{g} / \mathrm{ml}$ of AgNPs would approximately correspond to the total cellular deposition following 74 working weeks ( $8 \mathrm{~h}$ per day, 5 days per week) (21).

The production of reactive species, characteristic of the neutrophil oxidative burst, is initiated by the generation of superoxide anion radical $\left(\mathrm{O}_{2}^{-}\right)$through the activation of a multicomponent enzyme system, NADPH oxidase. This enzymatic complex is constituted by several subunits: Cytochrome b558, that is a heterodimer composed by two transmembrane proteins, gp91phox (phox: Phagocyte oxidase) and p22phox; and other four proteins, p47phox, p67phox, p40phox and Rac2, dispersed in cytosol. In neutrophils, NADPH oxidase occurs in a resting state, a primed state, a fully activated state and in a hyperactive state. In circulating neutrophils, NADPH oxidase occurs in a resting state, with the subunits distributed in the membrane and cytosol, ensuring a dormant inactivated state of the enzyme. The primed NADPH oxidase is a 'ready-to-go' state, which allows the enzyme to assemble all the subunits, gp91phox being able to use the cytosolic NADPH as the electron donor to reduce oxygen and produce $\mathrm{O}_{2}{ }^{-}$. The production of the first reactive specie indicate that the enzyme is fully activated $(22,23)$. Subsequently, other reactive species are produced, in a cascade form, as $\mathrm{H}_{2} \mathrm{O}_{2}$, which is concomitantly used, together with chlorine ions, by myeloperoxidase (MPO), a heme protein present in azurophil granules of neutrophils, to form hypochlorous acid ( $\mathrm{HOCl})(24)$. The information available in the literature about the effects of AgNPs on the human neutrophil oxidative burst is limited. For example, Poirier et al (14) described that citrate-coated AgNPs of 20 or $70 \mathrm{~nm}$ in size did not induce ROS production following exposure for $1 \mathrm{~h}$. In turn, the authors have previously described that $10 \mathrm{~nm}$ of PVP-coated AgNPs induced neutrophil oxidative burst, an effect not achieved by $50 \mathrm{~nm}$ AgNPs (15). In another 
A

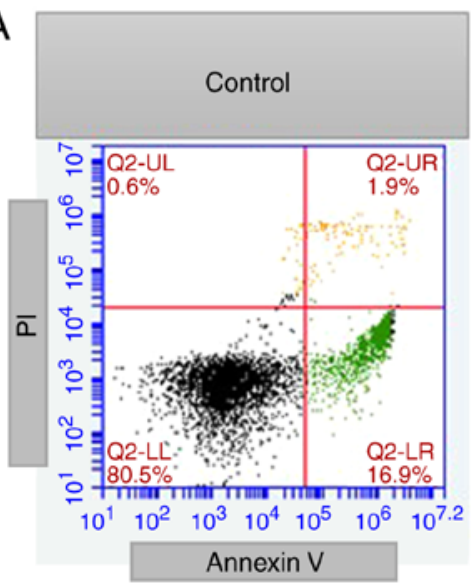

B

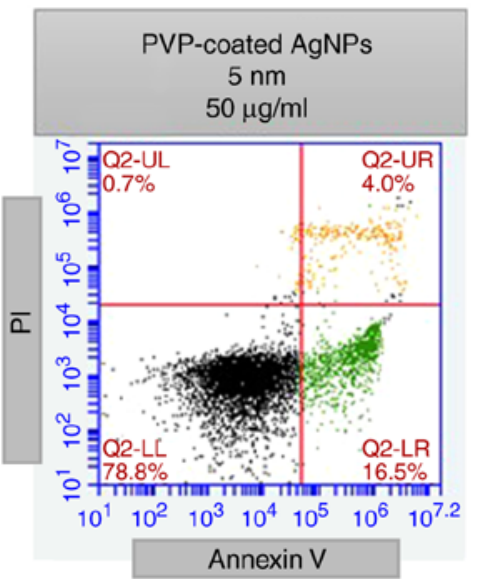

C

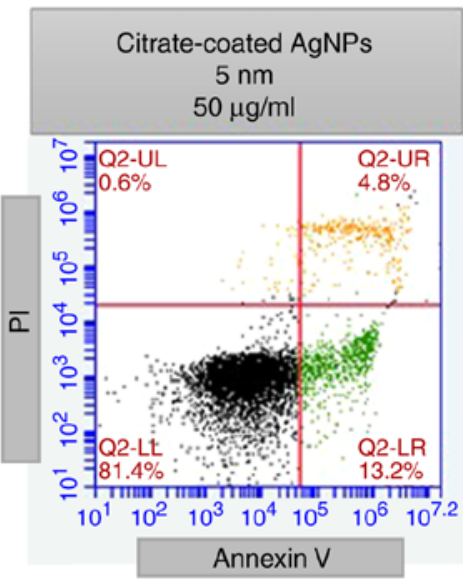

D

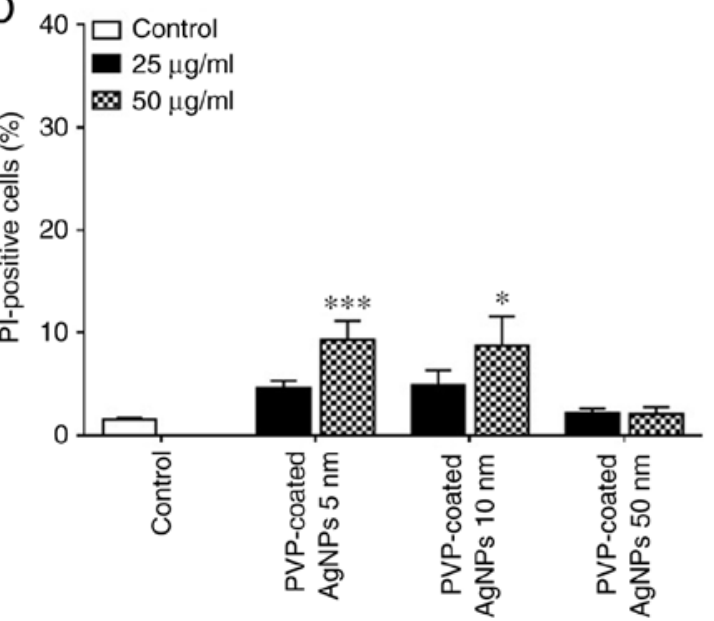

E 40 ㅁontrol

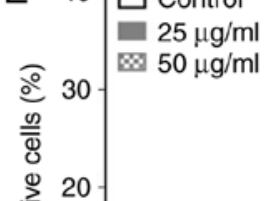

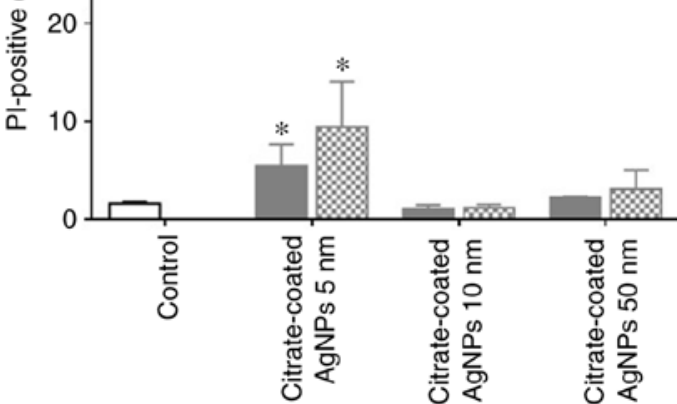

Figure 6. Representative flow cytometry plots of Annexin V [(Annexin V; $x$ axis)/PI (y axis)] binding assay of (A) control (without AgNPs), (B) $5 \mathrm{~nm}$ PVP-coated AgNPs, $50 \mu \mathrm{g} / \mathrm{ml}$, and (C) $5 \mathrm{~nm}$ citrate-coated AgNPs, $50 \mu \mathrm{g} / \mathrm{ml}$. Effect of (D) PVP-coated AgNPs and (E) citrate-coated AgNPs on human neutrophil necrosis, measured by PI. ${ }^{*} \mathrm{P}<0.05,{ }^{* * * *} \mathrm{P}<0.001$ when compared with the control (without AgNPs). Values are presented as the means $\pm \mathrm{SEM}(\mathrm{n} \geq 6$ ). PVP, polyvinylpyrrolidone; AgNPs, silver nanoparticles.

study involving neutrophils, it was reported that $20 \mathrm{~nm}$ AgNPs were more pro-inflammatory than $110 \mathrm{~nm}$ AgNPs in terms of neutrophil influx to the lungs, but not in terms of eosinophilic influx (9). These results suggest that the smallest-sized AgNPs induce a greater effect on human neutrophils. To investigate this hypothesis, the present study tested 3 sizes of AgNPs, 5, 10 and $50 \mathrm{nM}$, coated with PVP or citrate. For this purpose, DHR was used as a fluorescent probe. The results presented herein are in line with those previously described. The smallest-sized AgNPs induced a greater production of reactive species, irrespective of the coating agent used. It was previously demonstrated by the authors, through TEM images, that $10 \mathrm{~nm}$ of PVP-coated AgNPs were internalized in human neutrophils, and were located inside the cell, throughout the cytosol and also more intrinsically in phagosomes. AgNPs sized $50 \mathrm{~nm}$ were not able to enter cells, remaining outside the cells, close to the cellular membrane (15). In addition, Poirier et al (14) failed to detect the presence of citrate-coated AgNPs sized $70 \mathrm{~nm}$ inside human neutrophils. This difference in cellular localization could explain the size-dependent effect on human neutrophil activities. The so-called 'Trojan-horse' mechanism, in which nanoparticles are internalized within cells and then release high levels of toxic ions, has been proposed for AgNPs (25). In that sense, the present study examines the effect of the ion $\mathrm{Ag}^{+}$, through the analysis of $\mathrm{AgNO}_{3}$, and it was concluded that, under these experimental conditions, $\mathrm{Ag}^{+}$, per se, did not induce the neutrophil oxidative burst. This suggests that $\mathrm{Ag}^{+}$may not be the main factor contributing for the AgNP-induced activation of human neutrophils.

It is important to note that AgNPs coated with citrate triggered the most intensive response by neutrophils. PVP and citrate are frequently used coatings, essentially due to their low toxicity. PVP protects AgNPs from agglomerating by stabilizing the $\mathrm{Ag}^{+}$and $\mathrm{H}^{+}$in the suspension. The coordination of $\mathrm{Ag}^{+}$on the particle surface, with the $\mathrm{N}$ or $\mathrm{O}$ atoms of PVP, leads to the formation of a surface layer that inhibits agglomeration due to steric hindrance (26). By contrast, citrate is used in the form of citrate anions, which serve as a reducing agent, as well as to provide electrostatic repulsion, stabilizing the particles suspension $(27,28)$. To exclude any effect of the coating agent, PVP and citrate were tested per se, in the maximum proportion predictably existing in the AgNPs and no effect was detected on the neutrophil oxidative burst. As such, the effect seems to be due to the complex AgNPs coated with the capping agent.

It is currently accepted that PKC is implicated in the neutrophil oxidative burst, through the phosphorylation of p47 ${ }^{\text {phox }}$, resulting in the assembly and activation of phagocytic NADPH oxidase (29). Neutrophils contain 5 of the 11 known 
isoforms of PKC. These comprise 3 conventional isoforms designated $\alpha, \beta I$ and $\beta \mathrm{II}$, which are dependent on phosphatidylserine (PS), diacylglycerol (DAG) and calcium. There is also one novel isoform designated $\mathrm{PKCd}$, which also depends on phosphatidylserine and DAG, but is calcium-independent; and one atypical isoform designated $\mathrm{PKCz}$ which is DAG and calcium-independent but can be activated by phosphatidylserine, phosphatidic acid, or phosphatidylinositol. The expression of other isoforms, namely $\mathrm{PKCz}, \mathrm{PKCi} / 1$, and PKCq, remains a matter of debate (30). In the present study, a broad-spectrum PKC inhibitor was used and a decrease in the neutrophil oxidative burst, induced by the citrate- and PVP-coated AgNPs was observed. Despite the possible role of other kinases in the neutrophil activation, the obtained results indicate that the AgNPs induced the production of reactive species through the activation of PKC.

It has been proposed that calcium and DAG, both downstream products of phospholipase C (PLC), activate PKC, which in turn, completes a negative feedback loop by inhibiting PLC. Moreover, the classical PKC are distinguished from all other PKC as they require elevated calcium levels for maximal activity (31). In that sense, it was the authors' intention to determine whether AgNPs alter the intracellular calcium levels in human neutrophils. The results demonstrated that the citrate-coated AgNPs induced a significant increase in the intracellular calcium levels, which could explain the greater production of reactive species by these nanoparticles. By contrast, the PVP-coated AgNPs induced only a slight increase which was not statistically significant. The calcium movements in neutrophils can occur through a rapid emptying of the calcium stores, as endoplasmic reticulum, and then some intermediate mechanism translates this information to plasma membrane channels that enable calcium to entry and refill the depleted stores, in a process generally known as SOCE (17). Therefore, to determine the involvement of calcium in citrate-coated AgNP-induced neutrophil oxidative burst, ML-9, a SOCE broadly investigated inhibitor, was used and the production of reactive species was detected with DHR. The fluorescent signal decreased when ML-9 was used, revealing an important role of calcium in neutrophil activation by citrate-coated AgNPs.

The next step was to determine whether all the abovementioned events triggered by AgNPs and mostly by the citrate-coated AgNPs, interfere with neutrophil viability, focusing on apoptosis vs. necrosis. Apoptosis was detected by measuring the externalization of phosphatidylserine on the plasma membrane using fluorescent-tagged Annexin V. Under the tested experimental conditions, the number of Annexin V positive cells of treated or untreated cells did not differ significantly, suggesting that AgNPs did not induce neutrophil apoptosis.

PI, a popular red-fluorescent nuclear and chromosome counterstain was also used to analyze the effect of AgNPs on neutrophil viability. The necrotic effect was confirmed and the citrate-coated AgNPs induced higher levels of necrosis, since the smallest-sized AgNPs (5 nm) induced necrosis at lower concentrations, than the PVP-coated AgNPs. Flow cytometry provides information about the size and granularity of the cells, even without any specific marker. It was also observed that the citrate-coated AgNPs altered the morphology of the cells (data not shown) to a greater extent than the PVP-coated AgNPs. The reported cytotoxicity of AgNPs also varies among authors.
Nguyen et al (32) examined the cytotoxic effects of uncoated, PVP- and citrate-coated AgNPs (10, 50 and $75 \mathrm{~nm})$ on J774A.1 macrophages and HT29 epithelial cells. They concluded that the uncoated AgNPs were more cytotoxic than the coated AgNPs. Moreover, the PVP-coated AgNPs resulted in a greater loss of cell viability compared to the citrate-coated AgNPs, indicating a surface coating-dependent toxicity. By contrast, Wang et al (28) reported that PVP and citrate differentially affected the cytotoxicity of AgNPs on BEAS-2B cells, as demonstrated by the finding that PVP-coated AgNPs induced lower cytotoxicity, as was also found in the present study.

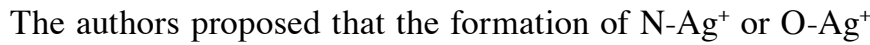
complexes by PVP results in reduced $\mathrm{Ag}^{+}$bioavailability. In addition, the authors of that study also stated that citrate lacks potent coordinating effects, and cannot provide protection against the cytotoxic effects of $\mathrm{Ag}^{+}$(28). This could explain the higher rates of cytotoxicity of citrate-coated AgNPs.

In conclusion, though the widespread use of AgNPs may indicate a good safety record, special attention is required to the extended exposure to these nanoparticles, considering its proinflammatory potential, which seems to increase as nanoparticles size decrease, specifically when citrate coating is used, as shown in the present study. It was demonstrated that AgNPs induced an increase in intracellular calcium levels, which are involved in the activation of PKC, resulting in the assembly of NADPH oxidase subunits with the subsequent production of reactive species. This excessive production of reactive species most probably contributes to the AgNP-induced decrease in neutrophil viability. The findings of the present study provide new insight into the interaction of AgNPs with human neutrophils, which is essential for the safer use of these nanoparticles.

\section{Acknowledgements}

The authors gratefully acknowledge Dr Margarida Amil and the nursing staff of the Centro Hospitalar do Porto-Hospital de Santo António blood bank for the collaboration in the recruitment of blood donors to participate in the study.

\section{Funding}

The present study was supported by UID/QUI/50006/2020 with funding from FCT/MCTES through national funds, and 'Programa Operacional Competitividade e Internacionalização' (COMPETE) (POCI-01-0145-FEDER-029248). DR and MF acknowledge the financial support from the European Union [FEDER funds through the Operational Competitiveness Program (COMPETE)] (POCI-01-0145-FEDER-029253 and POCI-01-0145-FEDER-029248, respectively).

\section{Availability of data and materials}

All data generated or analyzed during this study are included in this published article or are available from the corresponding author on reasonable request.

\section{Authors' contributions}

MF, FC and EF were involved in the conception and design of the study, and in the drafting of the manuscript. ML, AS, 
TS and DR performed all the experimental procedures, and contributed to the writing of the manuscript. All authors have read and approved the final manuscript.

\section{Ethics approval and consent to participate}

All patient-related procedures and protocols were performed in accordance with the Declaration of Helsinki and approved by the Ethics Committee of Centro Hospitalar do Porto. Written informed consent was obtained from all participants.

\section{Patient consent for publication}

Not applicable.

\section{Competing interests}

The authors declare that they have no competing interests.

\section{References}

1. Beyene HD, Werkneh AA, Bezabh HK and Ambaye TG: Synthesis paradigm and applications of silver nanoparticles (AgNPs), a review. Sustain Mater Technol 13: 18-23, 2017.

2. Vance ME, Kuiken T, Vejerano EP, McGinnis SP, Hochella MF Jr, Rejeski D and Hull MS: Nanotechnology in the real world: Redeveloping the nanomaterial consumer products inventory. Beilstein J Nanotechnol 6: 1769-1780, 2015.

3. Williams KM, Gokulan K, Cerniglia CE and Khare S: Size and dose dependent effects of silver nanoparticle exposure on intestinal permeability in an in vitro model of the human gut epithelium. J Nanobiotechnol 14: 62, 2016.

4. Bouwmeester H, Dekkers S, Noordam MY, Hagens WI, Bulder AS, de Heer C, ten Voorde SE, Wijnhoven SW, Marvin HJ and Sips AJ: Review of health safety aspects of nanotechnologies in food production. Regul Toxicol Pharmacol 53: 52-62, 2009.

5. Luo YH, Chang LW and Lin P: Metal-based nanoparticles and the immune system: Activation, inflammation, and potential applications. Biomed Res Int 2015: 143720, 2015.

6. Freitas M, Lima JL and Fernandes E: Optical probes for detection and quantification of neutrophils' oxidative burst. A review. Anal Chim Acta 649: 8-23, 2009.

7. Ribeiro D, Freitas M,Lima JL and Fernandes E: Proinflammatory pathways: The modulation by flavonoids. Med Chem Rev 35: 877-936, 2015.

8. Recordati C, De Maglie M, Bianchessi S, Argentiere S, Cella C, Mattiello S, Cubadda F, Aureli F, D'Amato M, Raggi A, et al: Tissue distribution and acute toxicity of silver after single intravenous administration in mice: Nano-specific and size-dependent effects. Part Fibre Toxicol 13: 12, 2016.

9. Seiffert J, Hussain F, Wiegman C, Li F, Bey L, Baker W, Porter A, Ryan MP, Chang Y, Gow A, et al: Pulmonary toxicity of instilled silver nanoparticles: Influence of size, coating and rat strain PLoS One 10: e0119726, 2015.

10. Park EJ, Bae E, Yi J, Kim Y, Choi K, Lee SH, Yoon J, Lee BC and Park K: Repeated-dose toxicity and inflammatory responses in mice by oral administration of silver nanoparticles. Environ Toxicol Pharmacol 30: 162-168, 2010.

11. Park EJ, Choi $\mathrm{K}$ and Park K: Induction of inflammatory responses and gene expression by intratracheal instillation of silver nanoparticles in mice. Arch Pharm Res 34: 299-307, 2011.

12. Alessandrini F, Vennemann A, Gschwendtner S, Neumann AU, Rothballer M, Seher T, Wimmer M, Kublik S, Traidl-Hoffmann C, Schloter M, et al: Pro-inflammatory versus immunomodulatory effects of silver nanoparticles in the lung: The critical role of dose, size and surface modification. Nanomaterials (Basel) 7: pii: E300, 2017.

13. Poirier M, Simard JC, Antoine F and Girard D: Interaction between silver nanoparticles of $20 \mathrm{~nm}$ (AgNP20) and human neutrophils: Induction of apoptosis and inhibition of de novo protein synthesis by AgNP20 aggregates. J Appl Toxicol 34: 404-412, 2014
14. Poirier M, Simard JC and Girard D: Silver nanoparticles of $70 \mathrm{~nm}$ and $20 \mathrm{~nm}$ affect differently the biology of human neutrophils. J Immunotoxicol 13: 375-385, 2016.

15. Soares T, Ribeiro D, Proenca C, Chisté RC, Fernandes E and Freitas M: Size-dependent cytotoxicity of silver nanoparticles in human neutrophils assessed by multiple analytical approaches. Life Sci 145: 247-254, 2016.

16. Freitas M, Porto G, Lima JL and Fernandes E: Isolation and activation of human neutrophils in vitro. The importance of the anticoagulant used during blood collection. Clin Biochem 41: $570-575,2008$

17. Ribeiro D, Freitas M, Rocha S, Lima J, Carvalho F and Fernandes E: Calcium pathways in human neutrophils-the extended effects of thapsigargin and ML-9. Cells 7: pii: E204, 2018.

18. Freitas M, Costa VM, Ribeiro D, Couto D, Porto G, Carvalho F and Fernandes E: Acetaminophen prevents oxidative burst and delays apoptosis in human neutrophils. Toxicol Lett 219: 170-177, 2013.

19. Ng LG, Ostuni R and Hidalgo A: Heterogeneity of neutrophils. Nat Rev Immunol 19: 255-265, 2019.

20. Silva RM, Anderson DS, Franzi LM, Peake JL, Edwards PC, Van Winkle LS and Pinkerton KE: Pulmonary effects of silver nanoparticle size, coating, and dose over time upon intratracheal instillation. Toxicol Sci 144: 151-162, 2015.

21. Gliga AR, Skoglund S, Wallinder IO, Fadeel B and Karlsson HL: Size-dependent cytotoxicity of silver nanoparticles in human lung cells: The role of cellular uptake, agglomeration and $\mathrm{Ag}$ release. Part Fibre Toxicol 11: 11, 2014.

22. Belambri SA, Rolas L, Raad H, Hurtado-Nedelec M, Dang PM and El-Benna J: NADPH oxidase activation in neutrophils: Role of the phosphorylation of its subunits. Eur J Clin Invest 48 (Suppl 2): e12951, 2018.

23. Babior BM: The leukocyte NADPH oxidase. Isr Med Assoc J 4: 1023-1024, 2002

24. Manda-Handzlik A and Demkow U: Neutrophils: The role of oxidative and nitrosative stress in health and disease. Adv Exp Med Biol 857: 51-60, 2015.

25. Hsiao IL, Hsieh YK, Wang CF, Chen IC and Huang YJ: Trojan-horse mechanism in the cellular uptake of silver nanoparticles verified by direct intra- and extracellular silver speciation analysis. Environ Sci Technol 49: 3813-3821, 2015.

26. Wang H, Qiao X, Chen J, Wang X and Ding S: Mechanisms of PVP in the preparation of silver nanoparticles. Mater Chem Phys 94: 449-453, 2005.

27. Henglein A and Giersig M: Formation of colloidal silver nanoparticles: Capping action of citrate. J Phys Chem B 103: 9533-9539, 1999.

28. Wang X, Ji Z, Chang CH, Zhang H, Wang M, Liao YP, Lin S, Meng H, Li R, Sun B, et al: Use of coated silver nanoparticles to understand the relationship of particle dissolution and bioavailability to cell and lung toxicological potential. Small 10: 385-398, 2014.

29. Cosentino-Gomes D, Rocco-Machado N and MeyerFernandes JR: Cell signaling through protein kinase $\mathrm{C}$ oxidation and activation. Int J Mol Sci 13: 10697-10721, 2012.

30. Bertram A and Ley K: Protein kinase C isoforms in neutrophil adhesion and activation. Arch Immunol Ther Exp (Warsz) 59: 79-87, 2011

31. Tintinger GR, Theron AJ, Steel HC, Cockeran R, Pretorius L and Anderson R: Protein kinase $\mathrm{C}$ promotes restoration of calcium homeostasis to platelet activating factor-stimulated human neutrophils by inhibition of phospholipase C. J Inflamm (Lond) 6: 29, 2009.

32. Nguyen KC, Seligy VL, Massarsky A, Moon TW, Rippstein P, Tan J and Tayabali AF: Comparison of toxicity of uncoated and coated silver nanoparticles. J Phys Conf Ser 429: 012025, 2013. 